\title{
Pathological Complete Response in Locally Advanced Breast Cancer after Neoadjuvant Chemotherapy: Survival Outcome and Its Relevance as a Surrogate End Point
}

\author{
Reshu Agarwal ${ }^{1} \quad$ U. G. Unnikrishnan ${ }^{2}$ Pavithran Keechilat ${ }^{3}$ Anupama Rajanbabu ${ }^{1}$ Wesley Jose ${ }^{3}$ \\ D. K. Vijaykumar ${ }^{1}$
}

\footnotetext{
${ }^{1}$ Department of Breast and Gynecology Oncology, Amrita Institute of Medical Sciences, Kochi, Kerala, India

2Biostatistics, Amrita Institute of Medical Sciences, Kochi, Kerala, India

${ }^{3}$ Medical Oncology, Amrita Institute of Medical Sciences, Kochi, Kerala, India
}

Address for correspondence D. K. Vijaykumar, MS, MCh, Department of Breast and Gynecology Oncology, Amrita Institute of Medical Sciences, Kochi, Kerala, India (e-mail: dkvijaykumar@aims.amrita.edu).

\section{South Asian J Cancer 2020;9:136-140.}

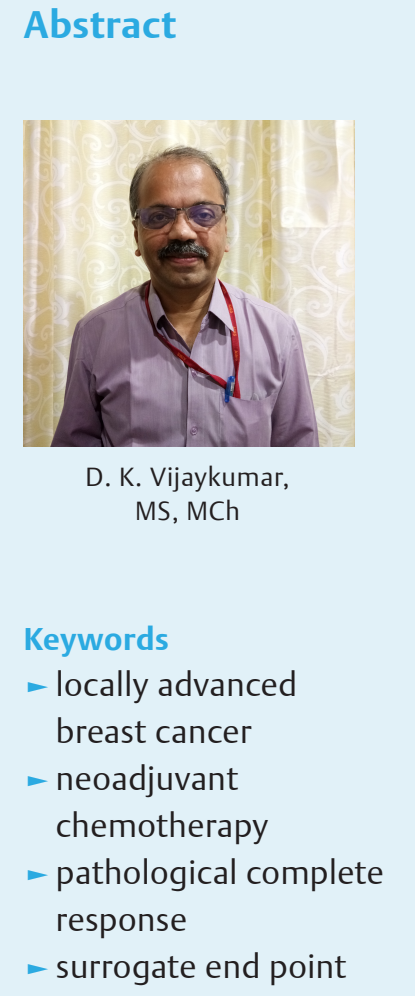

Background Pathological complete response ( $P C R$ ) to neoadjuvant chemotherapy has emerged as a reliable surrogate marker for improved survival in breast cancer (BC), but its role as a surrogate end point is still controversial.

Aims and Objectives The aim of the study is to investigate the clinical course of BC patients with $\mathrm{pCR}$ and to evaluate the relevance of $\mathrm{pCR}$ as a surrogate end point for survival.

Materials and Methods This was a single-institution retrospective analysis done at Amrita Institute of Medical Sciences. Records of BC patients from 2004 to 2014 were analyzed. Disease-free survival (DFS) and overall survival (OS) were compared using the Kaplan-Meier method and log-rank test, respectively. pCR and survival association were evaluated using regression analysis $\left(R^{2}\right)$.

Results Of 224 patients included in the study PCR rate was $15.2 \%$. The median duration of follow-up was 61 months (range: $3-151$ months). DFS (73.4 vs. $46.1 \%$, $p=0.032)$ and $O S(82.5$ vs. $56.4 \%, p=0.022)$ of $p C R$ cohort was significantly higher than non-pCR cohort. Recurrence rate was significantly lower in the $\mathrm{PCR}$ cohort at: All distant sites $(p=0.013)$, visceral sites $(p=0.007)$, both bone and visceral sites $(p=0.007)$, and nodal sites $(p=0.007)$. There was no difference in the bone-only recurrence $(p=0.315)$. Death rate was significantly lower in $\mathrm{pCR}$ cohort $(p=0.007)$. The $R 2$ value for $\mathrm{PCR}$ as a surrogate for DFS and OS was 0.006 and 0.004 , respectively. Conclusion $\mathrm{PCR}$ is a favorable prognostic factor associated with improved survival. However, there is no association between $\mathrm{PCR}$ and survival.

\section{Introduction}

Neoadjuvant chemotherapy (NAC) is considered as the standard of care in the management of locally advanced breast cancer (LABC). ${ }^{1}$ Pathological complete response (pCR) to NAC

How to cite this article: Agarwal R, Unnikrishnan UG, Keechilat $P$, Rajanbabu A, Jose W, Vijaykumar DK. Pathological Complete Response in Locally Advanced Breast Cancer after Neoadjuvant Chemotherapy: Survival Outcome and Its Relevance as a Surrogate End Point South Asian J Cancer 2020; 9(3):136-140.

DOI https://doi.org/10.1055/s-0040-1721238 ISSN 2278-330X. has emerged as a reliable surrogate marker for improved overall and disease-free survival (DFS) following BC diagnosis. $^{2-7}$ pCR is seen in $~ 15$ to $40 \%$ of BC patients receiving NAC. ${ }^{8}$ We aimed to investigate the clinical course of $\mathrm{BC}$ patients at our institution whose index tumor has exhibited
C 2020. Medlntel Services Pvt Ltd.

This is an open access article published by Thieme under the terms of the Creative Commons Attribution-NonDerivative-NonCommercial-License, permitting copying and reproduction so long as the original work is given appropriate credit. Contents may not be used for commercial purposes, or adapted, remixed, transformed or built upon. (https://creativecommons.org/licenses/by-nc-nd/4.0/) Thieme Medical and Scientific Publishers Pvt. Ltd., A-12, 2nd Floor, Sector 2, Noida-201301 UP, India 
pCR after NAC. We also aimed to evaluate the relevance of $\mathrm{pCR}$ as a surrogate end point for survival.

\section{Materials and Methods}

This is a single-institution, retrospective review of patients with LABC who underwent NAC followed by definitive surgery during the years 2004 to 2014 in the department of breast and gynecology oncology. The study was conducted after obtaining approval from the institutional review board.

pCR was defined as: No evidence of residual invasive malignancy in the breast or axilla; patients with only residual ductal carcinoma in situ following NAC were included in the PCR cohort.

All patients with histology proven nonmetastatic BC and information on the estrogen receptor (ER), progesterone receptor (PR), and human epidermal growth factor receptor-2 (HER2) status undergoing NAC were included in the study. All details of the initial staging, histology, type of surgery, and details of chemotherapy were collected.

The end points of the study were DFS and overall survival (OS).

\section{Statistical Analysis}

The descriptive statistics of treatment received, recurrence, morbidity, and mortality as of June 2017 are reported. The Kaplan-Meier method was used to calculate the survival rate and plot the survival curves. Differences in survival curves were examined using log-rank test. To evaluate the association between the $\mathrm{pCR}$ and the OS, regression analysis was performed. All analysis was performed using IBM SPSS for Windows (SPSS Inc, Chicago, Illinois, United States), version 20, and a $p$-value $<0.05$ was considered to indicate statistical significance.

\section{Results}

\section{Patient Characteristics}

A total of 4219 new BC cases were seen in the hospital during this time period (2004-2014). Excluding patients of early and metastatic $\mathrm{BC}$, those who came only for a second opinion or did not complete treatment at institution, those with incomplete treatment details available, and those who had concurrent chemoradiotherapy (RT) (as a part of another study), a total 224 patients met the inclusion criteria and hence were included in the analysis.

The mean age of diagnosis was 48.6 years (range: 25-81 years). Out of 224 patients, pCR was seen in 34 (15.2\% of NAC recipients) patients and non-pCR was seen in 190 (84.8\% of NAC recipients).

- Table 1 summarizes the comparison of patient-, tumor-, and treatment-specific characteristics between the $\mathrm{pCR}$ and non-pCR cohorts. Mean tumor size in both the cohorts (pCR $5.9 \mathrm{~cm}$ and non-pCR $6.2 \mathrm{~cm} ; p=0.601$ ) was comparable.

Triple-negative BC (TNBC) exhibited significantly higher pCR rate (pCR 64.7\% and non-pCR 41.1\%; $p=0.014$ ). However, hormone receptor-positive (HR+) BC exhibited significantly lower pCR rate (pCR 8.8\% and non-pCR 31.1\%; $p=0.006$ ). There was no difference in the $\mathrm{PCR}$ rate of HER2-enriched BC (pCR 26.5\% and non-pCR 27.8\%; $p=1.000$ ).

\section{Outcome}

The median duration of follow-up was 61 months (range: 3-151 months). Twenty-six patients (3 in pCR and 23 in non-pCR cohort) had follow-up of $<12$ months and without any event (these patients could not be contacted even after multiple attempts), hence excluded from the survival analysis. The DFS (73.4 vs. $46.1 \%, \log$ rank $p=0.032$ ) and OS (82.5 vs. $56.4 \%, \log$ rank $p=0.022$ ) of patients with $\mathrm{pCR}$ was significantly higher than non-pCR patients ( - Fig. 1A and B). The DFS of pCR patients at 5- and 10-year was 79.6 and $73.4 \%$, whereas of non-pCR patient was 57 and $46.1 \%$, respectively. The OS of pCR patients at 5- and 10-year was $89.4 \%$ and $82.5 \%$, whereas OS of non-pCR patient was 70.3 and $56.4 \%$, respectively.

A total of 80/198 (40.4\%) patients recurred during the study period. Significantly lower number of patients recurred in the pCR cohort (21.9\% in pCR and 44\% in non-pCR cohort; $p=0.013$ ). Recurrence at distant site was significantly lower in the $\mathrm{pCR}$ cohort (18.6\% in pCR and 39.8\% in non-pCR cohort; $p=0.013$ ). Bone only as a site of distant recurrence was not different in the two cohorts $(p=0.315$ ), but distant recurrence at visceral sites (12.5\% in pCR and 36.8\% in non-pCR cohort), both bone and visceral sites (nil in PCR and $10.2 \%$ in non-pCR cohort), and nodal site (nil in pCR and 3.6\% in non-pCR cohort) was significantly lower in pCR cohort ( - Table 2).

A total of 62/198 (31.3\%) patients died during the study period. BC-specific deaths were significantly lower in $\mathrm{pCR}$ cohort (12.5\% in pCR and 33.1\% in non-pCR cohort; $p=0.007$ ). Death due to Adriamycin-induced toxicity was seen in $4 / 198$ (2\%) of patients: 1 in pCR cohort and 3 in non-pCR cohort. Three $(1.8 \%)$ patients died due to other causes in non-pCR cohort (-Table 2).

\section{Linear Regression between Pathological Complete Response and Outcomes (Disease-Free Survival and Overall Survival)}

To evaluate the surrogacy of $\mathrm{pCR}$, the regression analysis was applied. The $R 2$ value for $\mathrm{PCR}$ as a surrogate for DFS was $0.006(p=0.294)$ and OS was $0.004(p=0.407)$ suggestive of minimal association between $\mathrm{PCR}$ and DFS and OS.

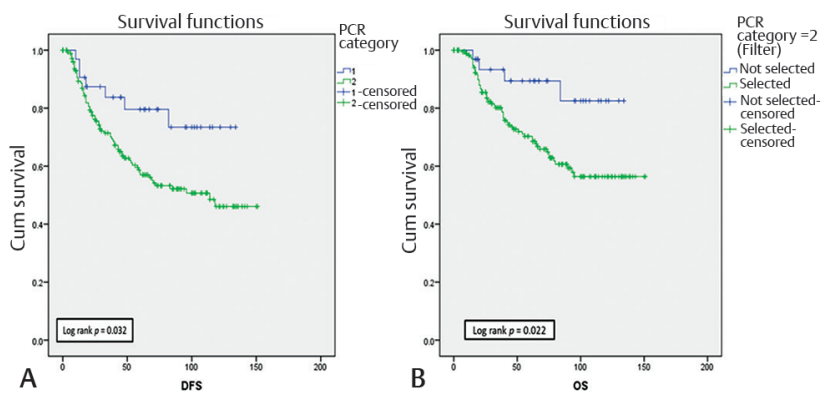

Fig. 1 Survival analysis in pathological complete response and nonpathological complete response cohort: (A) disease-free survival (DFS) (B) overall survival (OS). 
Table 1 Patient-, tumor-, and treatment-specific characteristics in pathological complete response and nonpathological complete response cohorts

\begin{tabular}{|c|c|c|c|}
\hline Variables & pCR $(n=34), n(\%)$ & Non-pCR $(n=190), n(\%)$ & $p$-Value \\
\hline \multicolumn{4}{|l|}{ Age $(y)$} \\
\hline Median (range) & $46(26-81)$ & $48.5(25-81)$ & 0.602 \\
\hline \multicolumn{4}{|l|}{ Menopausal status } \\
\hline Premenopausal & 19/34 (55.9) & $104 / 190(54.7)$ & \multirow[t]{2}{*}{1.000} \\
\hline Postmenopausal & $15 / 34(44.1)$ & $86 / 190(45.3)$ & \\
\hline \multicolumn{4}{|l|}{ T size $(\mathrm{cm})$} \\
\hline Mean (range) & $5.9(3-15)$ & $6.2(2-15)$ & 0.601 \\
\hline Intrinsic subtype & & & 0.012 \\
\hline Luminal & $3 / 34(8.8)$ & $59 / 190(31.1)$ & 0.006 \\
\hline HER2 enriched & $9 / 34(26.5)$ & $53 / 190(27.8)$ & 1.000 \\
\hline Triple negative & $22 / 34(64.7)$ & $78 / 190(41.1)$ & 0.014 \\
\hline \multicolumn{4}{|l|}{ Histopathological subtype } \\
\hline Ductal & $32 / 34(94.2)$ & 176/190 (92.6) & \multirow[t]{3}{*}{0.939} \\
\hline Lobular & $1 / 34(2.9)$ & $8 / 190(4.2)$ & \\
\hline Others & $1 / 34(2.9)$ & $6 / 190(3.2)$ & \\
\hline \multicolumn{4}{|l|}{ Type of NAC } \\
\hline Only anthracycline & $9 / 34(26.5)$ & $54 / 190(28.4)$ & \multirow[t]{3}{*}{0.877} \\
\hline Anthracycline + taxane & $23 / 34(67.6)$ & $121 / 190(63.7)$ & \\
\hline Others (CMF, only taxane) & $2 / 34(5.9)$ & $15 / 190(7.9)$ & \\
\hline \multicolumn{4}{|c|}{ Number of NAC cycles before surgery } \\
\hline$<50 \%$ cycles & $4 / 34(11.8)$ & $21 / 190(11.1)$ & \multirow[t]{3}{*}{0.893} \\
\hline$>50 \%$ cycles & 29/34 (85.3) & $160 / 190(84.2)$ & \\
\hline Complete $(6 / 8)$ & $1 / 34(2.9)$ & $9 / 190(4.7)$ & \\
\hline \multicolumn{4}{|l|}{ Type of surgery } \\
\hline Mastectomy & $31 / 34(91.1)$ & $170 / 190(89.5)$ & \multirow[t]{2}{*}{0.526} \\
\hline Breast conservation surgery & $3 / 34(8.8)$ & $20 / 190(10.5)$ & \\
\hline \multicolumn{4}{|l|}{ DCIS } \\
\hline Absent & 29/34 (85.3) & $62 / 190(32.6)$ & \multirow[t]{3}{*}{0.011} \\
\hline Present & $4 / 34(11.8)$ & $127 / 190(66.8)$ & \\
\hline Missing & $1 / 34(2.9)$ & $1 / 190(0.52)$ & \\
\hline
\end{tabular}

Abbreviations: CMF, cyclophosphamide, methotrexate, 5-fluorouracil regimen; DCIS, ductal carcinoma in situ; NAC, neoadjuvant chemotherapy; pCR, pathological complete response.

\section{Discussion}

It has been clearly established that a pCR at the time of surgery defined as ypTO ypNO or ypTO/is ypNO is associated with a favorable outcome in all patients who achieve it. ${ }^{9,10}$ pCR is seen in 15 to $40 \%$ of $\mathrm{BC}$ patients receiving NAC. ${ }^{8}$ Using the recommended definition of pCR (ypT0/ is ypN0), the pCR rate in the current study was $15.2 \%$ and achievement of PCR at the time of surgery was associated with the survival benefit.

The recurrence rate of $29 \%$ in pCR patients is almost identical to the rate of 13 to $25 \%$ reported in the literature.,11-14 The highest recurrence rate was observed in patients presented with advanced stage, $71.4 \%$ of recurred patients had cT3 and cT4, and all of them had lymph node metastasis. Dawood et al demonstrated that higher clinical stage was associated with worse outcomes even after achievement of PCR. ${ }^{15}$

The highest $\mathrm{PCR}$ rate in TNBC and lowest in HR+ tumors in the current study is in agreement with that reported in the literature. ${ }^{16,17}$ Our study did not show an equivalent $\mathrm{pCR}$ rate in HER2 enriched tumors owing to our inability to use anti-HER2 therapy due to cost concerns. von Minckwitz et al demonstrated that PCR appeared to be a reasonable surrogate end point for patients with luminal B/HER2-, ER/ PR/HER2+, and for TNBC, but not for those with luminal B/ HER2+ or luminal A tumors. ${ }^{16}$ However, in the current study, we found that out of 7 recurred patients: 4 had TNBC, 2 had ER/PR negative/HER2+ tumor, and 1 had ER/PR/HER2+. Thus, even for the subset of patients for whom pCR is a good prognosticator, its accuracy is not $100 \%$. 
Table 2 Outcome in pathological complete response and nonpathological complete response cohorts

\begin{tabular}{|c|c|c|c|}
\hline Variables & $\mathrm{pCR}(n=32), n(\%)$ & Non-pCR $(n=166), n(\%)$ & $p$-Value \\
\hline Overall recurrence & 7 (21.9) & $73(44)$ & \multirow[t]{3}{*}{0.013} \\
\hline Local recurrence & $1(3.1)$ & $7(4.2)$ & \\
\hline Distant recurrence & $6(18.6)$ & $66(39.8)$ & \\
\hline \multicolumn{4}{|l|}{ Site of distant recurrence } \\
\hline Bone only & $2(6.3)$ & $5(3)$ & 0.315 \\
\hline Visceral & $4(12.5)$ & $61(36.8)$ & 0.007 \\
\hline 1 site & $3(9.4)$ & $28(16.9)$ & \\
\hline$>1$ site & $1(3.1)$ & $10(6)$ & \\
\hline Bone + visceral & - & $17(10.2)$ & \\
\hline Nodal metastasis & - & $6(3.6)$ & \\
\hline Death & $4(12.5)$ & $58(34.9)$ & 0.007 \\
\hline Breast cancer specific death & $4(12.5)$ & $55(33.1)$ & \\
\hline Death due to other cause & - & $3(1.8)$ & \\
\hline
\end{tabular}

Abbreviation: pCR, pathological complete response.

We found that six out of seven patients recurred within 48 months ( $<5$ years) of diagnosis, out of which four recurred within 18 months. There was equivalent median survival of relapsed patients in pCR cohort (40 months) and non-pCR cohort (37 months). Similar observation was revealed by Fayanju et al in their study; they suggested that such a short interval between diagnosis and recurrence reflects failure of NAC in these patients despite achieving pCR. ${ }^{18}$

Cortazar et $\mathrm{al}^{9}$ and Berruti et $\mathrm{al}^{19}$ demonstrated minimal association between the effect of the treatment on pCR and the effect on event-free survival (EFS) and OS. They found that the $R 2$ values for $\mathrm{pCR}$ as a surrogate for EFS and OS were extremely low. NeoALTTO trial demonstrated the largest absolute improvement in $\mathrm{pCR}$ rate, but it failed to demonstrate the impact on survival. ${ }^{20}$ Consistent with the previously reported results, we found that the $R 2$ values for $\mathrm{pCR}$ as a surrogate for EFS and OS were extremely low at 0.006 and 0.004 , respectively.

Rose et al proposed three limitations of pCR to be used as a surrogate end point for improved survival. ${ }^{21}$ First, cancer is a micrometastatic systemic therapy, whereas $\mathrm{pCR}$ is a measure of effectiveness of the treatment only on the primary tumor. Short interval of recurrence and similar median survival of recurred patients in pCR and non-pCR cohort in the current study reflect the possibility of micrometastatic disease that continued to progress regardless of pCR. Second, effective treatment may not necessarily lead to increased pCR rate. In the current study, the treatment-specific characteristics were similar between the pCR and non-pCR cohort. Majority of the patients in both the cohorts received the standard anthracycline- and taxane-based regimen, with no difference in the timing of NAC and surgery. Third, additional pCRs achieved from an investigational therapy may simply occur in patients who would have been cured by standard treatment alone. In the current study, we did not find any difference in $\mathrm{PCR}$ rate of HER-2-enriched tumors because of the nonavailability of the standard anti-HER-2 treatment.
Our study has the limitations of being a single-institution retrospective analysis with small sample size, and the nonavailability of the grade of tumor, which is one of the most important prognosticators in $\mathrm{BC}$. Despite these limitations, to the best of our knowledge, this is the first Indian series where detailed analysis of the outcome of patients with PCR is done. Furthermore, our study is one of the few individual series where the issue of considering the $\mathrm{pCR}$ as a surrogate end point in clinical trials has been addressed. Our study might be helpful in adding evidence to the ongoing debate of use of $\mathrm{pCR}$ as an end point in clinical trials.

\section{Conclusion}

Thus, pCR is a favorable prognostic factor for individual patients undergoing treatment. pCR could be used as a marker that helps clinician in tailoring and reducing the intensity of subsequent treatment in patients with $\mathrm{pCR}$ as compared with non-pCR. However, its use as a surrogate end point in clinical trials is still a debatable issue. The current evidence, including the results of our study, does not demonstrate the strong association between the $\mathrm{pCR}$ and survival. Usage of $\mathrm{pCR}$ rate as an end point in clinical trials requires the establishment of strong association $\left(R^{2}>0.75\right)$ in prospective trial.

Funding

Nil.

Conflicts of Interest

There are no conflicts of interest.

\section{References}

1 Mauri D, Pavlidis N, Ioannidis JP. Neoadjuvant versus adjuvant systemic treatment in breast cancer: a meta-analysis. J Natl Cancer Inst 2005;97(3):188-194 
2 Gianni L, Eiermann W, Semiglazov V, et al. Neoadjuvant chemotherapy with trastuzumab followed by adjuvant trastuzumab versus neoadjuvant chemotherapy alone, in patients with HER2-positive locally advanced breast cancer (the NOAH trial): a randomised controlled superiority trial with a parallel HER2-negative cohort. Lancet 2010;375(9712) :377-384

3 Hutcheon AW, Heys SD, Sarkar TK; Aberdeen Breast Group. Neoadjuvant docetaxel in locally advanced breast cancer. Breast Cancer Res Treat 2003;79(1, Suppl 1) :S19-S24

4 Untch M, Möbus V, Kuhn W, et al. Intensive dose-dense compared with conventionally scheduled preoperative chemotherapy for high-risk primary breast cancer. J Clin Oncol 2009; 27(18):2938-2945

5 Kuerer HM, Newman LA, Smith TL, et al. Clinical course of breast cancer patients with complete pathologic primary tumor and axillary lymph node response to doxorubicin-based neoadjuvant chemotherapy. J Clin Oncol 1999;17(2):460-469

6 Rouzier R, Extra JM, Klijanienko J, et al. Incidence and prognostic significance of complete axillary downstaging after primary chemotherapy in breast cancer patients with T1 to T3 tumors and cytologically proven axillary metastatic lymph nodes. J Clin Oncol 2002;20(5):1304-1310

7 Esserman LJ, Berry DA, DeMichele A, et al. Pathologic complete response predicts recurrence-free survival more effectively by cancer subset: results from the I-SPY 1 TRIALCALGB 150007/150012, ACRIN 6657. J Clin Oncol 2012; 30(26):3242-3249

8 Kaufmann M, von Minckwitz G, Mamounas EP, et al. Recommendations from an international consensus conference on the current status and future of neoadjuvant systemic therapy in primary breast cancer. Ann Surg Oncol 2012; 19(5):1508-1516

9 Cortazar P, Zhang L, Untch M, et al. Pathological complete response and long-term clinical benefit in breast cancer: the CTNeoBC pooled analysis. Lancet 2014;384(9938) :164-172

10 Mazouni C, Peintinger F, Wan-Kau S, et al. Residual ductal carcinoma in situ in patients with complete eradication of invasive breast cancer after neoadjuvant chemotherapy does not adversely affect patient outcome. J Clin Oncol 2007; 25(19):2650-2655

11 Wolmark N, Wang J, Mamounas E, Bryant J, Fisher B. Preoperative chemotherapy in patients with operable breast cancer: nine-year results from National Surgical Adjuvant
Breast and Bowel Project B-18. J Natl Cancer Inst Monogr 2001; 30(30):96-102

12 Fisher B, Bryant J, Wolmark N, et al. Effect of preoperative chemotherapy on the outcome of women with operable breast cancer. J Clin Oncol 1998;16(8):2672-2685

13 Chollet P, Amat S, Cure H, et al. Prognostic significance of a complete pathological response after induction chemotherapy in operable breast cancer. Br J Cancer 2002;86(7):1041-1046

14 Gonzalez-Angulo AM, McGuire SE, Buchholz TA, et al. Factors predictive of distant metastases in patients with breast cancer who have a pathologic complete response after neoadjuvant chemotherapy. J Clin Oncol 2005;23(28):7098-7104

15 Dawood S, Broglio K, Kau SW, et al. Prognostic value of initial clinical disease stage after achieving pathological complete response. Oncologist 2008;13(1):6-15

16 von Minckwitz G, Untch M, Blohmer JU, et al. Definition and impact of pathologic complete response on prognosis after neoadjuvant chemotherapy in various intrinsic breast cancer subtypes. J Clin Oncol 2012;30(15):1796-1804

17 Bonnefoi $\mathrm{H}$, Litière $\mathrm{S}$, Piccart $\mathrm{M}$, et al; EORTC 10994/ BIG 1-00 Study investigators. Pathological complete response after neoadjuvant chemotherapy is an independent predictive factor irrespective of simplified breast cancer intrinsic subtypes: a landmark and two-step approach analyses from the EORTC 10994/BIG 1-00 phase III trial. Ann Oncol 2014;25(6):1128-1136

18 Fayanju OM, Nwaogu I, Jeffe DB, Margenthaler JA. Pathological complete response in breast cancer patients following neoadjuvant chemotherapy at a comprehensive cancer center: the natural history of an elusive prognosticator. Mol Clin Oncol 2015;3(4):775-780

19 Berruti A, Amoroso V, Gallo F, et al. Pathologic complete response as a potential surrogate for the clinical outcome in patients with breast cancer after neoadjuvant therapy: a meta-regression of 29 randomized prospective studies. J Clin Oncol 2014;32(34):3883-3891

20 Piccart-Gebhart M, Holmes E, Baselga J, et al. Adjuvant lapatinib and trastuzumab for early human epidermal growth factor receptor 2-positive breast cancer: results from the randomized phase III adjuvant lapatinib and/or trastuzumab treatment optimization trial. J Clin Oncol 2016;34(10):1034-1042

21 Rose BS, Winer EP, Mamon HJ. Perils of the pathologic complete response. J Clin Oncol 2016;34(33):3959-3962 